\title{
Evidence for the Volumetric Expansion of $N$-Benzylpyrazinium Hexafluoroantimonate and Epoxy Mixture during Curing Reaction
}

\author{
Youn Cheol $\mathrm{KIM}^{\dagger}$ and Jae-Rock LeE \\ Advanced Materials Division, Korea Research Institute of Chemical Technology, \\ P.O. Box 107, Yusong, Taejon 305-600, Korea
}

(Received April 21, 1998)

\begin{abstract}
Density measurements were used to obtain the direct evidence of the volumetric expansion during the polymerization of new catalytic ( $N$-benzylpyrazinium hexafluoroantimonate, BPH) epoxy thermosetting system. The density curve of the sample containing $0.5 \mathrm{wt} \% \mathrm{BPH}$ showed the independence of the reaction time, while the density of the sample containing $3.0 \mathrm{wt} \% \mathrm{BPH}$ increased with the reaction time at $150^{\circ} \mathrm{C}$. The volumetric expansion of $\mathrm{BPH} /$ epoxy mixture could be explained by the molecular packing owing to inter- and intramolecular hydrogen bonding. The FT-IR spectra of $3.0 \mathrm{wt} \%$ $\mathrm{BPH} /$ epoxy mixture showed an increase in the peak intensity of hydrogen-bonded hydroxyl group with the increase of reaction time at $150^{\circ} \mathrm{C}$. The concentration dependence on the volumetric expansion is well described that the curing process might lead to more network structure inducing changes in inter- and intramolecular hydrogen bonding at higher concentration of $\mathrm{BPH}$. KEY WORDS Volume Expansion / $N$-Benzylpyrazinium Hexafluoroantimonate / Epoxy / Hydrogen Bonding /
\end{abstract}

During the past several decades epoxy resins have been extensively studied on the advanced composites as matrices due to their excellent mechanical and thermal properties. However there is high shrinkage problem in the case of epoxy/amine system as it is widely used in various fields. In a fiber-reinforced composite, this shrinkage imposes some internal pressure onto the fiber so that physical bonding is enhanced. On the other hand, shrinkage in almost all epoxy resin systems is so high that it causes residual stress, warping, premature debonding of the fiber from the matrix, fiber buckling, and delamination in fiber-reinforced composite.

Extensive research has been carried out to reduce the problem of resin shrinkage. In order to improve shrinkage problem of epoxy resin, various adhesives were incorporated into the epoxy resins and some researches have been reported on the modification of curing conditions. Unfortunately there were no successful achievements to reduce the shrinkage problem. Since 1970s, Bailey et al. ${ }^{1}$ have studied on the introducing a series of spiroortho compounds that expand upon curing reaction. There are some problems, such as complicated synthesis procedures and low glass transition temperature $\left(T_{\mathrm{g}}\right)$.

In the early 1980 s, focused on long term stability and electrical properties, an increasing aspect has been studied on the cationic epoxy curing. ${ }^{2-5}$ Crivello $^{2}$ and AbuAbdoun $^{3}$ reported that the triarylsulfonium salts and benzyl phosphonium salts can serve as photo-induced cationic initiators in the polymerization of epoxy resins. And it has been proved by Pappers et al. ${ }^{4}$ and Endo et al. ${ }^{5}$ that several sulfonium salts act as thermally latent cationic initiator in the curing reaction of epoxy resins. Gu et al. ${ }^{6}$ and Morio et al. ${ }^{7}$ also reported that the aliphatic sulfonium salts can be also excellent thermally latent initiators for epoxy resins without any co-initiator. Lee et $a l^{8}{ }^{8}$ developed a new class of Lewis acid catalysts, 1,2-substituted-1,1-dimethylhydrazinium hexafluoroantimonates. As reported in our previous paper, ${ }^{9}$ it was proved that this salt served as thermal latent cationic initiator in the cure reaction of diglycidylether bisphenol A (DGEBA) having the volumetric expansion upon curing.

However, the cause for the expansion is not well understood. Recently Ishida and Low ${ }^{10}$ proposed that the volumetric expansion of the benzoxazine-based phenolic resin is mostly due to the consequence of molecular packing influenced by inter- and intramolecular hydrogen bonding.

In order to investigate the reason of the volumetric expansion of $N$-benzylpyrazinium hexafluoroantimonate (BPH)/epoxy system, we synthesized new BPH catalysts with $N$-benzyl group containing pyridium salt and measured the evidence of the volumetric expansion and the change of hydroxyl peaks upon curing using density measurements and FT-IR.

\section{EXPERIMENTAL}

\section{Materials}

Epoxy resin used in this study was DGEBA (YD 128 supplied from Kukdo Chem. Co. of Korea). Epoxide equivalent weight of the DGEBA was $185-190 \mathrm{~g} \mathrm{eq}^{-1}$.

BPH catalyst were synthesized according to the procedure of Lee $e t a l{ }^{8}$ and the purity and the chemical structures of product were confirmed by the elemental analysis, FT-IR, and the ${ }^{1} \mathrm{H}$ NMR. ${ }^{9}$

Various compositional epoxy mixtures were prepared from DGEBA and BPH as following procedures: the required amount of catalyst (BPH) was accurately weighed into a $250 \mathrm{ml}$ beaker equipped with mechanical stirrer and was dissolved in acetone. And then the moderate amount of epoxy (DGEBA) was added. The beaker containing the sample was stirred for $10 \mathrm{~min}$

\footnotetext{
${ }^{\dagger}$ To whom all correspondence should be addressed.

Present address: Department of Materials Science \& Engineering, University of Illinois at Urbana-Champaign, 1304 W Green Street, Urbana, Illinois 61801, U.S.A.
} 
and degassed for $60 \mathrm{~min}$ before testing. The catalyst concentrations for the used composition correspond to DGEBA/BPH ratios of 99.5:0.5, 97:3, and 95:5. DGEBA and BPH chemical structures are shown in Figure 1.

\section{Measurements}

Isothermal DSC measurements were carried out with Du Pont DSC 910 supported by a Du Pont thermal analyzer. The amount of sample was about $10-15 \mathrm{mg}$.

The FT-IR instrument used was a Hartmann \& Brawn Model Bomen MB 102 Spectrophotometer. The scans were from 400 to $4000 \mathrm{~cm}^{-1}$ and required $40 \mathrm{~s}$ to complete. Since the scans were conducted at high temperatures, a Grasedy-Specac Hot Cell was used to mount the samples. A Grasedy-Specac Temperature Controller (Model Eurotherm) was used along with heating elements and a thermocouple to control the temperature of the hot cell. Maximum 20 scans per run were carried out.

Density was used to evaluate the expansion of the cured materials. Density measurements were performed according to ASTM D792 (Method A) for all materials.

\section{RESULTS AND DISCUSSION}

As reported in our previous paper, ${ }^{9}$ DGEBA/BPH systems showed more than one type of reaction and $\mathrm{BPH}$ can be thermally excellent latent initiator for epoxy resins without any co-initiator. For the DGEBA/BPH systems the initiation temperatures were about $150^{\circ} \mathrm{C}$. Therefore, all isothermal experiments were performed at $150^{\circ} \mathrm{C}$. Figure 2 shows isothermal DSC thermograms for the DGEBA containing $0.5\left(\mathrm{~S}_{05}\right)$ and $3 \mathrm{wt} \%\left(\mathrm{~S}_{30}\right)$ of $\mathrm{BPH}$ at $150^{\circ} \mathrm{C}$. In case of the $\mathrm{S}_{05}$ sample, the isothermal DSC curve showed the decrease in the exothermic peak up to $180 \mathrm{~min}$ at $150^{\circ} \mathrm{C}$. On the other hand the $\mathrm{S}_{30}$ sample almost finished its reaction within $60 \mathrm{~min}$ at $150^{\circ} \mathrm{C}$. These

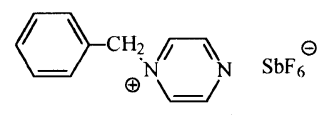

Chemical Structure of BPH

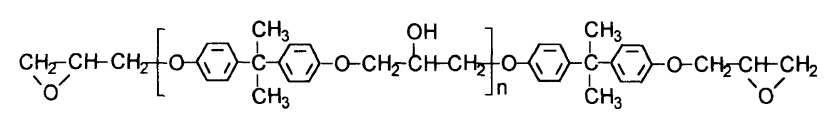

Chemical Structure of DGEBA

Figure 1. The chemical structure of the DGEBA and BPH. results indicate, as the concentration of $\mathrm{BPH}$ increases, that the availability of the radical to react with the epoxides in the initial stage of cuve would be more. The thermal and curing properties of DGEBA/BPH systems are summarized in Table I.

Figure 3 shows the density measured at room temperature as a function of reaction time at $150^{\circ} \mathrm{C}$. The $\mathrm{S}_{05}$ sample shows no change in room temperature density as the reaction proceeds, while $S_{30}$ and $S_{50}$ show a slight decrease in the density measured at room temperature with the reaction time at $150^{\circ} \mathrm{C}$ indicating an evidence of volumetric expansion. In case of most cure systems the volumetric shrinkage occurs commonly during curing process because molecules move from a van der Waals distance of separation to a covalent distance of separation. Small molecules are sometimes released as byproducts during the polymerization process, thus further reducing the volume in resulting polymer. When the polymerization reaction is completed, the material is cooled from the curing temperature which is usually at an elevated temperature with thermal shrinkage occurring.

Ishida and Low ${ }^{10}$ reported that the curing reaction of benzoxazine-based phenolic resin results in a decrease in the density measured at room temperature. They proposed that the volumetric expansion of the benzoxazine-based phenolic resin is mostly due to the consequence of the molecular packing influenced by inter-

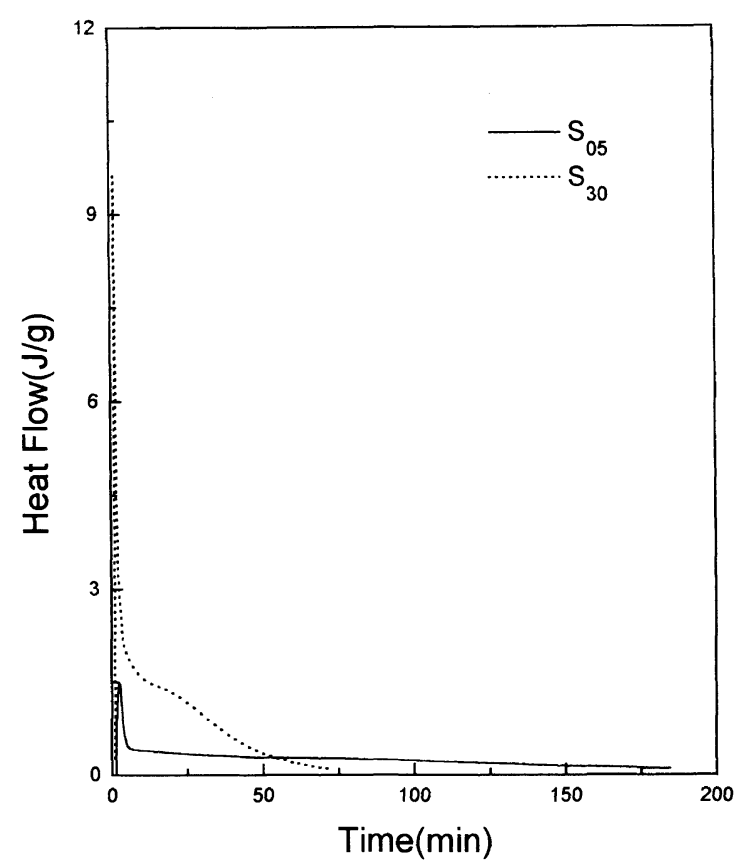

Figure 2. Isothermal DSC thermograms at $150^{\circ} \mathrm{C}$.

Table 1. Thermal and curing properties of DGEBA/BPH systems

\begin{tabular}{|c|c|c|c|c|c|c|c|c|c|}
\hline \multicolumn{2}{|c|}{ Samples } & \multirow{2}{*}{\begin{tabular}{|l}
$T_{\mathrm{g}}^{\mathrm{a}}$ \\
${ }^{\circ} \mathrm{C}$
\end{tabular}} & \multicolumn{3}{|c|}{ DSC peaks $/{ }^{\circ} \mathrm{C}$} & \multirow{2}{*}{$\begin{array}{c}\text { Total heat } \\
\text { of reaction } / \mathrm{Jg}^{-1}\end{array}$} & \multicolumn{3}{|c|}{ Gelation time at $150^{\circ} \mathrm{C} / \mathrm{min}$} \\
\hline ID & $\mathrm{wt} \%$ of $\mathrm{BPH}$ & & $1 \mathrm{st}$ & 2nd & $3 \mathrm{rd}$ & & Epoxide $^{b}$ & $\mathrm{DSC}^{\mathrm{c}}$ & $G^{\prime} / G^{\prime \prime}=1^{\mathrm{d}}$ \\
\hline $\mathrm{S}_{05}$ & 0.5 & - & 160.9 & - & 214.3 & 165.9 & 20.1 & 19.7 & 17.1 \\
\hline $\mathrm{S}_{30}$ & 3 & 127.4 & 150.6 & 163.4 & 202.6 & 463.3 & 8.6 & 9.1 & 8.2 \\
\hline $\mathrm{S}_{50}$ & 5 & 134.1 & 151.3 & 158.2 & 194.9 & 490.6 & 3.7 & 4.1 & 3.6 \\
\hline
\end{tabular}

${ }^{\mathrm{a}}$ Glass transition temperatures were obtained from second run of DSC. ${ }^{\mathrm{b}}$ Time when conversion is 0.6. Conversion was measured from the disappearance of the peak at $913 \mathrm{~cm}^{-1}$ base on $\mathrm{C}-\mathrm{H}$ stretch at $1510 \mathrm{~cm}^{-1}$. ${ }^{\mathrm{c}}$ Time when conversion is 0.6 . Conversion was measured from the isothermal DSC curve. ' Time when the ratio of dynamic moduli is 1 . Here, $G^{\prime}$ means storage modulus and $G^{\prime \prime}$ means loss modulus. 


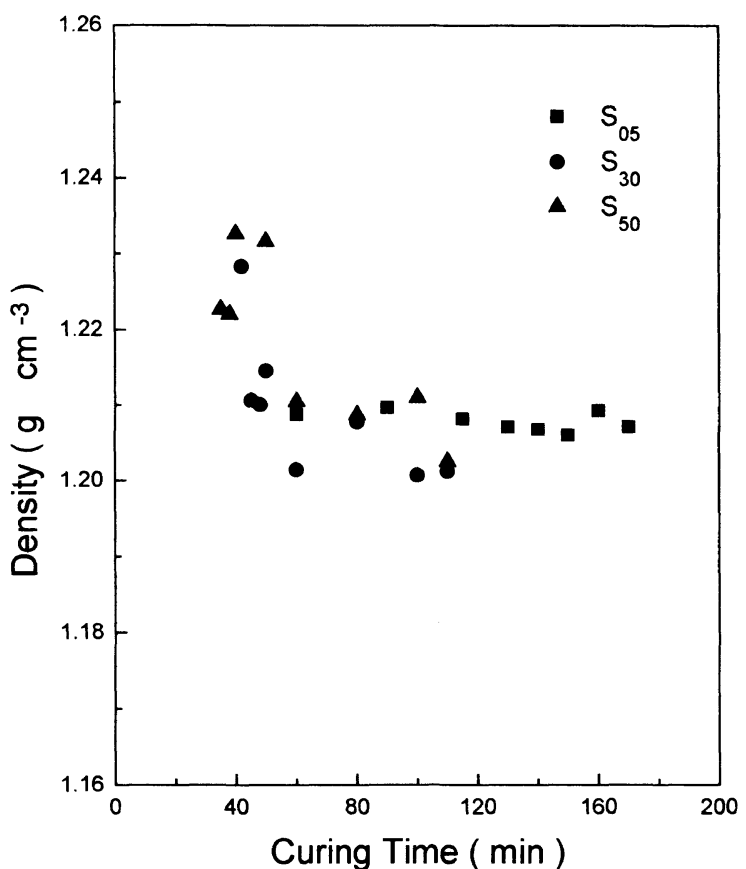

Figure 3. The change of room temperature density as a function of reaction time.

and intramolecular hydrogen bonding. For the DGEBA/ BHP systems an intuitive explanation for the variation of the density with curing time and the BPH compositions is that the hydrogen bond configuration was influenced on the molecular packing property. As reported on our previous paper, ${ }^{9}$ the gelation of DGEBA/BPH system begins with the initiation reaction between epoxides and BPH or between hydroxyl groups in the epoxy resins and BPH. In case of the $\mathrm{S}_{30}$ sample, the radical to react with the hydroxyl groups within the epoxy resins in the initial stage would be more available than that of $\mathrm{S}_{05}$ sample, and the increase in the availability would result in the increase in network formation. The proximity of adjacent molecules by the network formation increases the possibility of inter- and intramolecular hydrogen bonding. That is, when the hydroxyl proton forms hydrogen bonds with an adjacent hydroxyl group, the molecular packing structure can alter toward increasing unoccupied volume (free volume) in the network structure. ${ }^{10}$ The unoccupied volume would cause to hinder the molecular packing, which decreases eventually the density of cured material.

In order to verify this assumption, FT-IR spectroscopic measurements were carried out in the hydroxyl group of the DGEBA/BPH systems as a function of reaction time at $150^{\circ} \mathrm{C}$. Before discussing the FT-IR results, it is first necessary to assign the hydrogenbonded hydroxyl groups. There have been a large number of investigations for thermosetting resins. ${ }^{10,11-14}$ $\mathrm{Wu}$ et al. ${ }^{14}$ reported that three hydroxyl peaks exist for the hydrogen bond formation between novolac type phenolic resin and polyhydroxyl ether of bisphenol A. According to their results, the absorption of free hydroxyl group appears at the region $3650-3500 \mathrm{~cm}^{-1}$, while two hydrogen-bonded hydroxyl groups (dimer and multimer) shows a broad absorption band at the region $3550-3300 \mathrm{~cm}^{-1}$. Ishida and $\mathrm{Low}^{10}$ reported also that hydrogen-bonded hydroxyl groups, from both inter- and

Polym. J., Vol. 30, No. 11, 1998
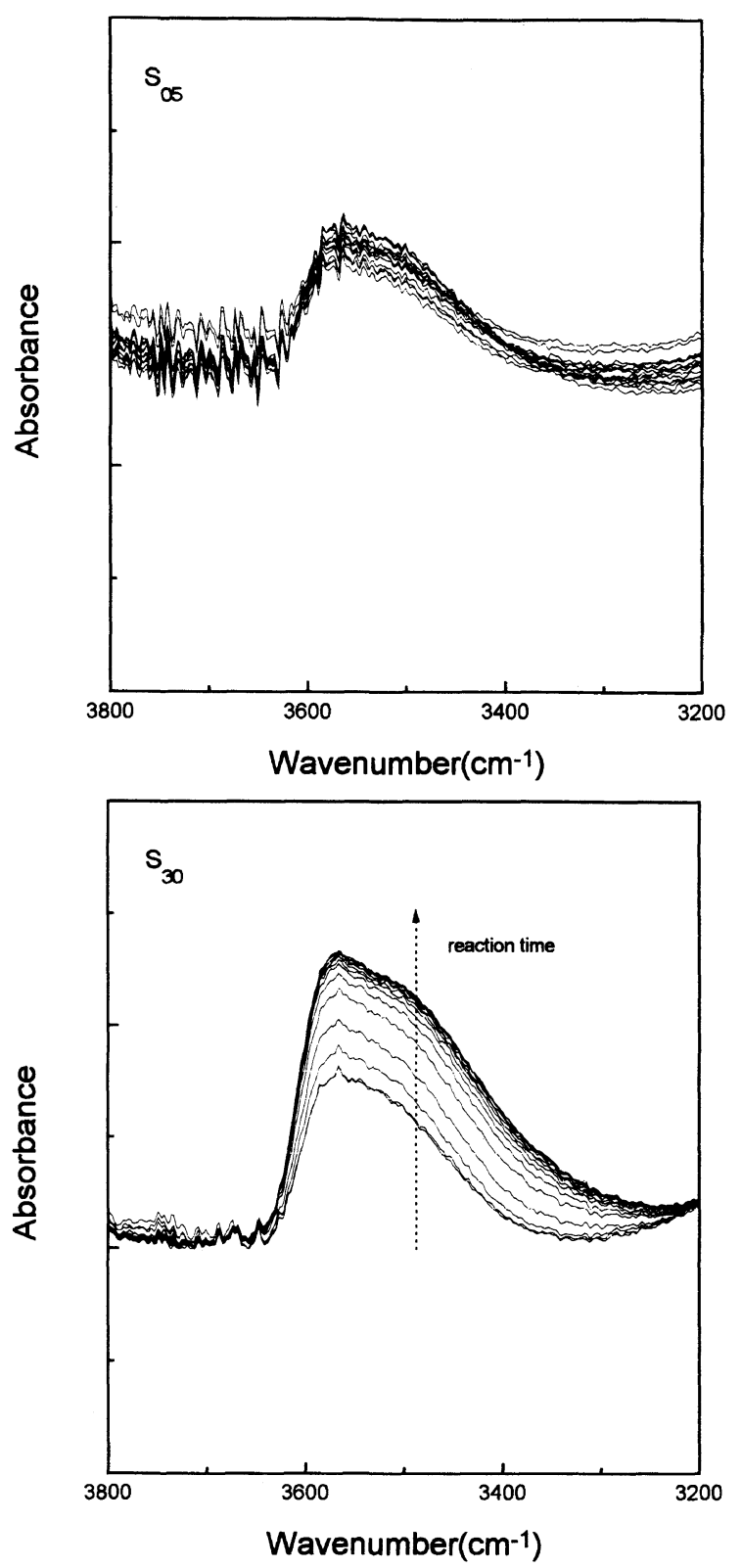

Figure 4. Change of FT-IR spectra of $\mathrm{S}_{05}$ and $\mathrm{S}_{30}$ at $150^{\circ} \mathrm{C}$ with reaction time.

intramolecular interactions, appear between 3600 and $2500 \mathrm{~cm}^{-1}$. For the epoxy/amine system Wang and Gillham $^{12}$ reported that intramolecular hydrogenbonded hydroxyl group appears at $3581 \mathrm{~cm}^{-1}$, whereas intermolecular hydrogen-bonded hydroxyl group appears at $3430 \mathrm{~cm}^{-1}$.

Figure 4 shows the infrared spectra of DGEBA/BPH systems during the progress of curing at $150^{\circ} \mathrm{C}$. For the hydroxyl peak of the $S_{05}$ sample, the peak intensity is nearly constant during curing reaction. Based on our assumption, no change in FT-IR spectra of hydroxyl group is indicative of no change in the density measured at room temperature. As shown in Figure 3 there was no detectable change in the density of the $\mathrm{S}_{05}$ sample agreed to the FT-IR results. On the other hand there is a big change in the peak intensity of hydroxyl group for the $\mathrm{S}_{30}$ sample. Even though the relative hydrogenbonded interactions could not be determined precisely, the hydroxyl bands around $3600-3300 \mathrm{~cm}^{-1}$ for the curing samples appear to be broader and stronger than 

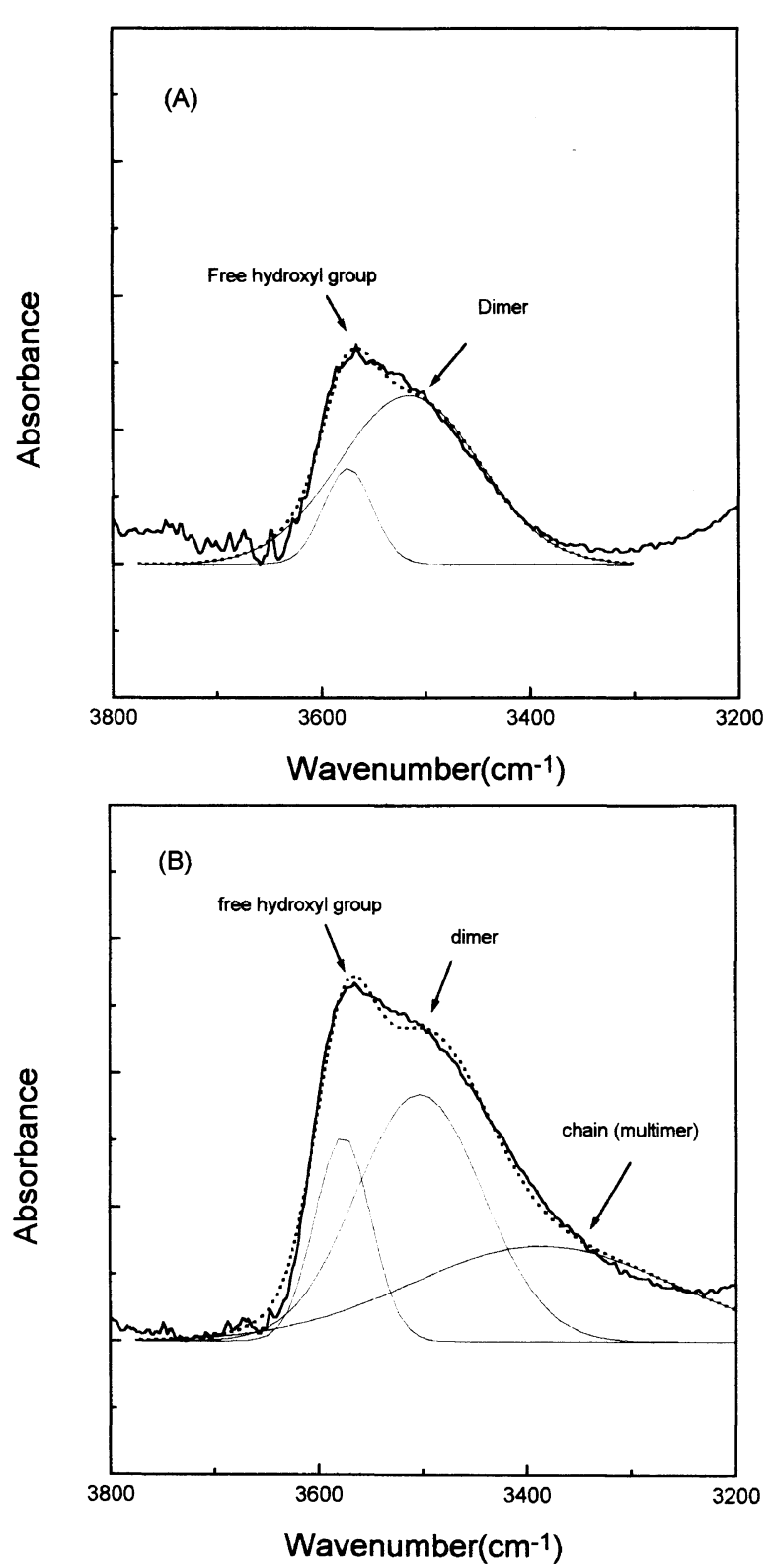

Figure 5. FT-IR spectra of $\mathrm{S}_{30}$ at $150^{\circ} \mathrm{C}$. (A) just after starting the reaction and $(B)$ after curing for $60 \mathrm{~min}$.

that of the initial state of the $S_{30}$ mixture at $150^{\circ} \mathrm{C}$. These results suggest that the molecular interactions by hydrogen bonding have a significant effect on the density since the density of a curing material is a direct consequence of the change of molecular packing.

To evaluate precisely the relative hydrogen-bonded hydroxyl group from the FT-IR spectra shown in Figure 4 , an iterative least-square computer program was used to obtain the best fit of the recorded spectrum in this region $\left(3800\right.$ to $3200 \mathrm{~cm}^{-1}$ ). It was confirmed that there are three Gaussian peaks in the hydroxyl stretching region of the $S_{30}$ sample after curing reaction for 60 min at $150^{\circ} \mathrm{C}$. However, the $\mathrm{S}_{30}$ sample just after starting the reaction displays only two Gaussian peaks in the hydroxyl stretching region.

$\mathrm{Wu}$ et $a{ }^{14}{ }^{14}$ reported that, in addition to the free hydroxyl group absorption $\left(3650-3580 \mathrm{~cm}^{-1}\right)$, the hydrogen-bonded hydroxyl band could be further resolved into a 'dimer' hydrogen-bonded hydroxyl peak at 3430$3550 \mathrm{~cm}^{-1}$ and the 'multimer' hydrogen-bonded hy- droxyl peak around $3250-3380 \mathrm{~cm}^{-1}$.

Returning to our FT-IR results as shown in Figure 5, at initial stage of curing at $150^{\circ} \mathrm{C}$ two peaks are centered at 3575 and $3515 \mathrm{~cm}^{-1}$, which are contributed by the free hydroxyl group and dimer hydrogen-bonded hydroxyl group, respectively. After curing for $60 \mathrm{~min}$ at $150^{\circ} \mathrm{C}$, one peak is centered at $3577 \mathrm{~cm}^{-1}$, which is contributed by the free hydroxyl group. The other broad band is observed centered between 3503 and $3386 \mathrm{~cm}^{-1}$, which is attributed to a wide distribution of dimer and multimer hydrogen-bonded hydroxyl stretching, respectively. These results suggest that intra- and intermolecular hydrogen bonding resulting from dimer and multimer formation results in the change of molecular packing, and it takes place volumetric expansion of DGEBA/BPH systems.

For the composition dependence on volumetric expansion, high concentration of $\mathrm{BPH}$ increases the possibility of inter- and intramolecular hydrogen bonding of hydroxyl group due to the proximity of adjacent molecules and it influences on the volumetric expansion of DGEBA/BPH mixture. That is, it can be interpreted that the composition dependence for volumetric expansion is related to the structural change based on the initiation reaction and the network formation during curing reaction with increasing, of $\mathrm{BPH}$ concentration as reported previously. ${ }^{9}$

\section{CONCLUSION}

For the $\mathrm{S}_{30}$ and $\mathrm{S}_{50}$ samples the density measured at room temperature indicates occurring volumetric expansion during curing reaction at $150^{\circ} \mathrm{C}$. The volumetric expansion of DGEBA/BPH systems upon curing is related to a change of the molecular packing. The interand intramolecular hydrogen bonding of hydroxyl group can influence on the change of molecular packing. The hydrogen bonding of hydroxyl group was confirmed by FT-IR spectra measured at $150^{\circ} \mathrm{C}$. Owing to the composition dependence of initiation reaction and network formation, the volumetric expansion of DGEBA/BPH system depends on the BPH concentration.

\section{REFERENCES}

1. W. J. Bailey, Polym. Prepr., 18, 17 (1977).

2. J. V. Crivello and J. L. Lee, Macromolecules, 14, 1141 (1981).

3. I. I. Abu-Abdoun and A. Ali, Eur. Polym. J., 29, 1439 (1993).

4. S. P. Pappas and L. H. Hill, J. Coat. Technol., 53, 43 (1981).

5. T. Endo, A. Kikkawa, H. Uno, and H. Sato, J. Polym. Sci., Polym. Lett. Ed., 27, 73 (1989).

6. J. Gu, S. C. Narang, and E. M. Pearce, J. Appl. Polym. Sci., 30, 2997 (1985).

7. K. Morio, H. Murase, and H. Tsuchiya, J. Appl. Polym. Sci., 32, 5727 (1986).

8. S. B. Lee, Y. S. Park, K. W. Lee, and T. Endo, Chem. Lett., 17, 287 (1995).

9. Y. C. Kim, S. J. Park, and J. R. Lee, Polym. J., 29, 759 (1997).

10. H. Ishida and H. Y. Low, Macromolecules, 30, 1099 (1997).

11. V. Bellenger and Verdu, Polymer, 28, 1079 (1987).

12. X. Wang and J. K. Gillham, J. Appl. Polym. Sci., 43, 2267 (1991).

13. T. O. Skourlis and R. L. Mccullough, J. Appl. Polym. Sci., 62, 481 (1996).

14. H. D. Wu, C-C. M. Ma, and P. P. Chu, Polymer, 38, 5419 (1997). 